DOI: 10.1002/adma.((please add manuscript number))

\title{
A luminescent molecular thermometer for long-term absolute temperature measurements at the nanoscale
}

By Carlos D. S. Brites, Patricia P. Lima, Nuno J. O. Silva, Angel Millán, Vitor S. Amaral, Fernando Palacio* and Luís D. Carlos*

Keywords: Luminescent molecular thermometer, Hybrid Materials, Magnetic Nanoparticles

[*] Mr. C. D. S. Brites, Dr. P. P. Lima, Dr. N. J. O. Silva, Prof. V. S. Amaral, Prof. L. D. Carlos Departamento de Física and CICECO, Universidade de Aveiro 3810-193 Aveiro (Portugal)

E-mail: 1carlos@ua.pt

Dr. A. Millán, Prof. F. Palacio

Departamento de Fisica de la Materia Condensada

Facultad de Ciencias and Instituto de Ciencia de Materiales de Aragón, CSIC-Universidad de Zaragoza

50009 Zaragoza (Spain)

E-mail:palacio@unizar.es 


\section{Submitted to

Temperature is a fundamental thermodynamic variable, the measurement of which is crucial in countless scientific investigations and technological developments, accounting at present for $75 \%-80 \%$ of the sensor market throughout the world. ${ }^{[1]}$ The traditional liquid-filled and bimetallic thermometers, the thermocouples, the pyrometers and the thermistors are generally not suitable for temperature measurements at scales below $10 \mu \mathrm{m}$. This intrinsic limitation has encouraged the development of new noncontact accurate thermometers with micrometric and nanometric precision, a challenging research topic increasingly hankered for. ${ }^{[2-9]}$

Current developments ${ }^{[4]}$ of thermometers operating below a few micrometers and with high spatial resolution include: Ga-filled carbon/MnO nanotubes, ${ }^{[2]}$ temperature dependent conformation modifications of molecular spring superstructures, ${ }^{[3]} \operatorname{Raman}^{[10]}$ and infrared ${ }^{[11]}$ spectroscopic probing, scanning thermal microscopy probes, ${ }^{[6]}$ and luminescence-based measurements. ${ }^{[5-9,12-29]}$

Nanotube based systems require a transmission electron microscope for the read-out and calibration, an evident limitation for practical applications. Infrared observation is restricted by diffraction resolution and other spectroscopic methods (such as Raman), and scanning microscopy systems have a read-out rate limited by material surface conditions and probe motion (typically $30 \mathrm{~ms}$ per pixel, ${ }^{[29]}$ making real-time temperature mapping unfeasible). By contrast, the thermal dependence of phosphor (organic dyes or trivalent lanthanides, $\mathrm{Ln}^{3+}$ ) luminescence is a noninvasive and accurate alternative technique that works remotely by way of an optical detection system, even in biological fluids, strong electromagnetic fields and fast-moving objects. Among the luminescent thermometers proposed so far only a handful of them illustrate effectively the temperature sensing/mapping at a submicrometer scale: a cellular thermometer with an organic dye as the temperature probe, ${ }^{[8]}$ a scanning thermal microscope with 
$\mathrm{Er}^{3+} / \mathrm{Yb}^{3+}$ co-doped fluoride glass small particles ${ }^{[6]}$ or $\mathrm{PbF}_{2}$ nanoparticles ${ }^{[29]}$ glued at the tip extremity; 2D distribution maps using ${ }^{5} \mathrm{D}_{0}$ rise-time temporal response in $\mathrm{Y}_{2} \mathrm{O}_{3}: \mathrm{Eu}^{[26]}$ and siloxane hybrid nanoparticles (size ranging from 20 to $30 \mathrm{~nm}$ ) incorporating a $\mathrm{Eu}^{3+} \operatorname{tris}(\beta$-diketonate $)$ complex. ${ }^{[9]}$

Here, we report the development of a unique $\mathrm{Eu}^{3+} / \mathrm{Tb}^{3+}$ luminescent nanothermometer ${ }^{[30]}$ combining a number of properties, which make it a step forward relatively to the single $\mathrm{Ln}^{3+}$-based devices proposed so far. This new thermometer is self-referencing, allowing absolute measurements in the $10-350 \mathrm{~K}$ temperature range. Its temperature sensitivity is up to $4.9 \% \cdot \mathrm{K}^{-1}, 1.5$ times larger than the highest value reported previously ${ }^{[9]}$, and it exhibits high photostability in long-term use. The variation of the $\mathrm{Eu}^{3+} / \mathrm{Tb}^{3+}$ ratio affords tunability to the temperature working range. Alternatively, tunability is also accomplished by changing the host matrix, thus modifying the interaction between the $\mathrm{Ln}^{3+}$ and the host matrix energy levels. To obtain a high resolution 2-dimensional temperature mapping, a judicious choice of the host matrix enables processing the thermometer material as a film. Finally, the combination of the molecular thermometer with a nanometric magnetic/luminescent host matrix provides the device multifunctionality at the nanoscale.

The present luminescent molecular thermometer consists of $\left[\mathrm{Eu}(\mathrm{btfa})_{3}(\mathrm{MeOH})(\right.$ bpeta $\left.)\right]$ and $\left[\mathrm{Tb}(\mathrm{btfa})_{3}(\mathrm{MeOH})(\right.$ bpeta $\left.)\right] \beta$-diketonate chelates $^{[30]}$ (Fig. S1a in the Supporting Information) embedded into organic-inorganic hybrid nanoclusters $(100-400 \mathrm{~nm})$ formed by a maghemite $\left(\gamma-\mathrm{Fe}_{2} \mathrm{O}_{3}\right)$ magnetic core (hydrodynamic size of $21.0 \pm 4.0 \mathrm{~nm}$, Fig. 1a) coated with a tetraethyl orthosilicate/aminopropyltriethoxysilane (TEOS/APTES) organosilica shell (see the experimental section for details about synthesis and labeling). The hybrid host was rationally designed in order to present an excited triplet state with energy slightly above 
that of the $\mathrm{Tb}^{3+5} \mathrm{D}_{4}$ emitting state, thus warranting the occurrence of thermally-driven ${ }^{5} \mathrm{D}_{4} \rightarrow$ host energy transfer and, consequently, the determination of the temperature dependence of the ${ }^{5} \mathrm{D}_{4}$ emission. As the energy difference between that triplet state and the $\mathrm{Eu}^{3+}{ }^{5} \mathrm{D}_{0}$ emitting level is too large to permit thermally-driven depopulation, the $\mathrm{Tb}^{3+} / \mathrm{Eu}^{3+}$ relative intensity guarantees the absolute measurement of temperature, with spatial resolution adjustable by the size of the nanoclusters to which the luminescent probes are anchored. We remark that similar host-to- $\mathrm{Ln}^{3+}$ energy resonance conditions may be obtained using other $\beta$-diketonate chelates with different ligands. The selfcalibration and the temperature measurement by way of relative intensities (an inexpensive, much easier and less time-consuming method than the measurements of the emission quantum yield and the excited state lifetime) overcome the well-known drawbacks of intensity-based measurements, such as variation of the sensor concentration and drifts of the optoelectronic system. ${ }^{[9]}$

The multifunctional nanoparticles are dispersible in water and show a bimodal size distribution centered at 120 and $390 \mathrm{~nm}$ as seen in DLS measurements (Fig. 1a). Nanoparticles with these characteristic sizes are also observed in SEM and TEM images (Figs. 1 b,c and Figure S2 in the Supporting Information). Aggregation of the nanoparticles occur during evaporation of the dispersions on the sample holder, with these particles showing regions with high and low contrast, corresponding to maghemite $/ \mathrm{Eu}^{3+} / \mathrm{Tb}^{3+}$ cores and to the siliceous matrix, respectively (Fig. 1c). EDS mappings show $\mathrm{Eu}^{3+}$ and $\mathrm{Tb}^{3+}$ distributions with contours and shapes similar to those of the nanoparticles (Fig. 1d), indicating that most of the nanoparticles contain both $\mathrm{Eu}^{3+}$ and $\mathrm{Tb}^{3+}$. The magnetic properties of the $\gamma-\mathrm{Fe}_{2} \mathrm{O}_{3} @$ TEOS/APTES nanoparticles reveal superparamagnetic behaviour above 200 K (Figure S3 in the Supporting Information). 
In optical microscopy observations, the $\mathrm{Eu}^{3+} / \mathrm{Tb}^{3+}$ co-doped $\gamma-\mathrm{Fe}_{2} \mathrm{O}_{3} @$ TEOS/APTES nanoparticles appear as red spots with sizes of the order of $5 \mu \mathrm{m}$, as illustrated in Fig 1e for the NP3-1.3 nanoparticles (see experimental section for sample labeling), with each spot corresponding to the emission either of a nanoparticle or a nanoparticle aggregate the red color being the result of the dominance of the $\mathrm{Eu}^{3+}$ emission at roomtemperature (Figs. S4 and S5 in the Supporting Information). Since the emission color is a measure of the absolute temperature - as it is shown below - the use of this luminescent molecular thermometer coupled with an optical microscope constitutes a thermometer with micrometer spatial resolution. The larger spot size as compared to the nanoparticles aggregates is direct consequence of the limited space resolution of the instrument.

The temperature dependence of the emission spectra $(14-300 \mathrm{~K})$ of the co-doped $\mathrm{Eu}, \mathrm{Tb} \gamma-\mathrm{Fe}_{2} \mathrm{O}_{3} @$ TEOS/APTES NP3 nanoparticles is illustrated in Fig. 2a (and in Figs. S6 and S7 in the Supporting Information), and whereas the intensity of the green $\mathrm{Tb}^{3+}$ luminescence strongly decreases as the temperature increases, the intensity of the red $\mathrm{Eu}^{3+}$ lines starts to increase at precisely the same temperature at which the $\mathrm{Tb}^{3+}$ emission for NP3-1.1, NP3-1.2 and NP3-1.3 starts to decrease, remaining approximately constant for NP3-1.10 (see experimental section for sample labeling). As this temperature dependence - appropriately illustrated by the calculus of the Commission Internacionale d'Éclairage (CIE) (x,y) color coordinates (Fig. 2b) strongly depends on the relative proportion of the $\mathbf{1}$ and $\mathbf{2}$ complexes anchored at the surface of the nanoparticles (Figs. S6 and S7 in the Supporting Information), the ability to fine-tune the nanocomposites emission from the green area towards the red region of Fig. $2 b$ as a function of the temperature is readily managed by changing the relative proportion of embedded complexes. The temperature dependence of the $\mathrm{Eu}^{3+}$ emission 
is rationalized assuming a concerted two-step process involving only the Boltzmann energy factor $\Delta E$. First, the excited triplet state of the TEOS/APTES layer with energy above that of the ${ }^{5} \mathrm{D}_{4}$ emitting state is populated through thermally-driven ${ }^{5} \mathrm{D}_{4}$-to-host energy transfer (diminishing, therefore, the ${ }^{5} \mathrm{D}_{4} \rightarrow{ }^{7} \mathrm{~F}_{5}$ intensity). Then, the triplet host level transfers a fraction of the absorbed energy to $\mathrm{Eu}^{3+}$ ions (through the multipolar and exchange mechanisms $\left.{ }^{[31]}\right)$. For diluted $\mathrm{Eu}^{3+}$ nanoparticles, the probability of the host-to$\mathrm{Eu}^{3+}$ energy transfer mechanism should be strongly reduced (due to a larger average $\mathrm{Tb}^{3+}-\mathrm{Eu}^{3+}$ distance) resulting in that the ${ }^{5} \mathrm{D}_{0} \rightarrow{ }^{7} \mathrm{~F}_{2}$ intensity should be practically temperature independent up to $250-275 \mathrm{~K}$. This is exactly what is observed for NP31.10 (Fig. S7 in the Supporting Information), thus bearing out the above interpretation.

The temperature dependence of the ${ }^{5} \mathrm{D}_{0}$ and ${ }^{5} \mathrm{D}_{4}$ lifetimes is shown in Fig. S8 in the Supporting Information and in Fig. 2c, respectively (with the corresponding decay curves being well modeled by single exponential functions; Fig. S9 in the Supporting Information). Whereas the ${ }^{5} \mathrm{D}_{0}$ lifetime remains approximately constant $\left(0.53-0.75 \times 10^{-3}\right.$ s, Fig. S8 in the Supporting Information, excluding, therefore, $\mathrm{Tb}^{3+}$-to-Eu ${ }^{3+}$ energy transfer ${ }^{[32]}$ ), the ${ }^{5} \mathrm{D}_{4}$ lifetime decreases approximately one order of magnitude, (Fig. 2c), a behavior that can be understood considering the presence of a thermally activated nonradiative mechanism associated to the emitting centers of the TEOS/APTES layer, whose energy is centered at $\sim 22500 \pm 1500 \mathrm{~cm}^{-1}$ (Fig. S10 in the Supporting Information), in accord with the one previously reported for aminopropylsilica nanospheres annealed at $673 \mathrm{~K} \cdot{ }^{[33,34]}$ The non-radiative de-excitation probability of the $\mathrm{Tb}^{3+5} \mathrm{D}_{4}$ level $\left(20590 \pm 5 \mathrm{~cm}^{-1}\right)$ may be approximately described by the Mott-Seitz model, which expresses the temperature dependence of the experimental lifetime as: ${ }^{[32]}$ 


$$
\tau^{-1}=\tau_{r}^{-1}+k \exp \left(-\frac{\Delta E}{k_{g} T}\right)
$$

where $\tau_{r}$ is the radiative lifetime, $k$ is the migration energy rate, $\Delta E$ is the activation energy between the triplet host level and the ${ }^{5} \mathrm{D}_{4}$ state, $k_{B}$ is the Boltzmann constant and $T$ is the absolute temperature. The activation energies obtained for NP3-1.1, NP3-1.2 and NP3-1.3 are $615 \pm 19,521 \pm 18$ and $687 \pm 14 \mathrm{~cm}^{-1}$, respectively, in agreement with the above suggestion that, for these nanoparticles, the thermally activated non-radiative mechanism involves the emitting levels of the TEOS/APTES layer.

The absolute temperature measurement is inferred defining an experimental parameter $\Delta\left(\Delta=I_{E u}^{2}-I_{T \zeta}^{2}\right)$, related to the integrated areas $I_{T b}$ and $I_{E u}$ of the ${ }^{5} \mathrm{D}_{4} \rightarrow{ }^{7} \mathrm{~F}_{5}$ $\left(I_{T b}\right.$ at $\left.545 \mathrm{~nm}\right)$ and the ${ }^{5} \mathrm{D}_{0} \rightarrow{ }^{7} \mathrm{~F}_{2}\left(I_{E u}\right.$ at $\left.612 \mathrm{~nm}\right)$ transitions. Figure 3a shows the temperature dependence of $\Delta$ for the NP3 nanoparticles, while Fig. $3 \mathrm{~b}$ presents the temperature dependence of the sensitivity (defined in the experimental section) as a function of the relative $\mathrm{Tb}^{3+}$-to- $\mathrm{Eu}^{3+}$ ratio. The best value $\left(4.9 \% \cdot \mathrm{K}^{-1}\right)$ is the largest one measured so far for molecular thermometers, being 1.5 times larger than the highest value previously reported using the same intensity ratio technique. ${ }^{[9]}$ Setting, as quality limit, a minimum sensitivity value of $1 \% \cdot \mathrm{K}^{-1}$, leads to an unprecedent optimal operation range of $\sim 60-80 \mathrm{~K}$ around the temperature of maximum sensitivity (120-190 K). Furthermore, the absolute emission quantum yields of the NP3 nanoparticles - ranging from $0.20 \pm 0.02$ (NP3-1.3) to $0.37 \pm 0.04$ (NP3-1.2) (Table S1 in the Supporting Information) - are sufficiently high so as to permit the use of commercial fiber optics for excitation and detection.

To make absolute measurements at higher temperatures feasible, the activation energy $\Delta E$ must be increased, which can accomplished either by increasing the 


\section{Submitted to

TEOS/APTES ratio $^{[33]}$ or by designing a different host matrix presenting an excited triplet state located at higher energy, relatively to that of the TEOS/APTES layer. Here, we report this latter approach using $\mathrm{Eu}^{3+} / \mathrm{Tb}^{3+}$ co-doped di-ureasils ${ }^{[35,36]}$ (allowing the processability of the thermometer as films), whose excitation and emission spectra are shown in Fig. $\mathrm{S} 11$ in the Supporting Information. Increasing $\Delta E$ to $\approx 2000 \mathrm{~cm}^{-1}$ (as calculated using Eq. 1), the thermometer range of operation is now shifted to room temperature (Figs. 3b,c). The magnetic properties of the $\gamma-\mathrm{Fe}_{2} \mathrm{O}_{3}$ nanoparticles embedded into the di-ureasil host (U1.3) also display superparamagnetism above $200 \mathrm{~K}$ (Figure S3 in the Supporting Information). The maximum absolute emission quantum yield and the maximum sensitivity of $\mathbf{U 1 . 3}$ are $0.16 \pm 0.02$ (Table S1 in the Supporting Information) and $1.9 \% \cdot \mathrm{K}^{-1}$ (at $201 \mathrm{~K}$, Fig. 3b), respectively. The $\mathrm{Eu}^{3+} / \mathrm{Tb}^{3+}$ co-doped diureasils are, therefore, sufficiently sensitive to be used in the physiological temperature range, as illustrated by the calibration curve of $\mathbf{U} 1.3$ between $298-328 \mathrm{~K}$ (Fig. S12b in the Supporting Information).

A demonstration of the spatial resolution of the $\mathrm{Eu}^{3+} / \mathrm{Tb}^{3+}$ thermometer was performed mapping the temperature of an integrated circuit covered with a U1.3 layer (Figs. 4a,b). An optical fiber is used to excite and collect the hybrid emission which is converted in absolute temperature using the $\mathbf{U}-\mathbf{1 . 3}$ calibration curve. A map of the temperature of the circuit was also recorded with a state-of-the-art commercial IR camera (see the supporting information for details). The comparison between the two mappings is illustrated in Figs. 4c,d showing a better spatial resolution for the $\mathrm{Eu}^{3+} / \mathrm{Tb}^{3+}$ thermometer, despite the $450 \mu \mathrm{m}$ of the optical fiber's inner diameter being $\sim 2.5$ times larger than the camera pixel size. In our experiment, the spatial resolution achieved, 35 $\mu \mathrm{m}$ (Fig. 4d), is estimated by the minimal distance resolved by the thermometer, when the temperature changes above the temperature uncertainty $(0.5 \mathrm{~K}$ in this case; 


\section{Submitted to

Supporting Information). This procedure shows, therefore, that the thermometer proposed here permits measuring absolute temperatures in regions with high temperature gradients (line B in Fig. 4b), as well as in regions presenting complex temperature distributions (line A in Fig. 4b). We draw attention to the fact that although the spatial resolution of the thermometer obviously depends upon the spatial resolution of the optical detector $(\approx 1 \mu \mathrm{m}$ in the case of the optical microscope in Fig. 1e, for example), the ultimate resolution limit of the thermometer is not reached yet, since the thermographic technique used in this experiment can - with appropriate sensing/mapping devices - detect temperature variations in nanosized regions.

In summary, the luminescent molecular thermometer introduced here combines: i) ability to fine-tune emission color as a function of temperature and $\mathrm{Eu}^{3+} / \mathrm{Tb}^{3+}$ proportion; ii) self-referencing that allows absolute measurements; iii) $4.9 \% \cdot \mathrm{K}^{-1}$ maximum temperature sensitivity (better than $0.5 \% \cdot \mathrm{K}^{-1}$ in the physiological temperature range); iv) high photostability for long-term use; v) flexibility to be processed as thin films for sensing/mapping large areas with a spatial resolution limited by the size of the optical detectors $(\sim 1-10 \mu \mathrm{m}$ for commercial optical fibers and CCD cameras); vi) a temperature uncertainty of 0.5 degree; and vii) multifunctionality as it can be hosted in silica-coated magnetic nanoparticles. When compared this thermometer with the $\mathrm{Ln}^{3+}$ based ones proposed so far it clearly represents a step forward in thermometry at the nanoscale. We anticipate that the synergetic outcomes arising by combining temperature sensing/mapping and superparamagnetism opens the way for new exciting applications, especially in the biomedical field. In particular, such association will provide a unique instrument to map, in a non-invasive way, temperature distributions in biological tissues (e.g., in tumors) during heat release, due to the application of an ac field to magnetic 


\section{ADVANCED}

nanoparticles (magnetic hyperthermia ${ }^{[37]}$ ), this being, with no doubt, a powerful tool for the study of biochemical micro-processes occurring within a cell. 


\section{Submitted to

\section{Experimental}

Synthesis of $\mathrm{Ln}^{3+}$-containing $\gamma-\mathrm{Fe}_{2} \mathrm{O}_{3} @$ TEOS/APTES nanoparticles. The $\mathrm{Ln}^{3+}$ containing $\gamma-\mathrm{Fe}_{2} \mathrm{O}_{3} @$ TEOS/APTES nanoparticles $(\mathrm{Ln}=\mathrm{Eu}$, Tb or Eu/Tb), named as NP1, NP2 and NP3, respectively, were prepared following the procedure described in detail in the Supporting Information. $\mathrm{Eu}, \mathrm{Tb}$ co-doped nanoparticles were produced using Eu:Tb ratios of 2:1, 1:1, 1:2, 1:3 and 1:10, named as NP3-2.1, NP3-1.1, NP3-1.2, NP3-1.3 and NP3-1.10, respectively. The corresponding ferrofluid was prepared by dispersing nanoparticle powders in water by sonication.

Synthesis of the Di-ureasil hybrid sensing film. The first step of the synthesis (detailed in the Supporting Information) involves the formation of a urea cross-linked organic-inorganic hybrid precursor $^{[35]}$ (Fig. S1b in the Supporting Information). In the second step, the $\left[\mathrm{Eu}(\mathrm{btfa})_{3}(\mathrm{MetOH})(\right.$ bpeta $\left.)\right]$ (1) and $\left[\mathrm{Tb}(\mathrm{btfa})_{3}(\mathrm{MetOH})(\right.$ bpeta $\left.)\right]$ (2) complexes were incorporated as ethanolic solutions together with water and $\mathrm{HCl}$ for promoting the hydrolysis of the alkoxysilane precursor. The sample, named $\mathbf{U}-\mathbf{1 . 3}$, was prepared as a thick $(\sim 10 \mu \mathrm{m})$ film, aged for seven days at $318 \mathrm{~K}$.

Characterization. The morphology, chemical composition and size distribution of the particles were characterized by scanning and transmission electron microscopy and dynamic light scattering (see the Supporting Information for details). Optical microscopy was carried out on a Carl Zeiss Axiovert $200 \mathrm{M}$ fluorescence microscope equipped with Apotome, using a $365 \mathrm{~nm}$ excitation wavelength. The samples were prepared by dispersing the NP3 powders in water by sonication, dropping this dispersion in a microscope slide and drying it in open air. Emission spectra were recorded on a Fluorolog- $3{ }^{\circledR}$ 2-Triax, Horiba Scientific, with a modular double grating excitation spectrometer and a TRIAX 320 single-emission monochromator coupled to a R928 Hamamatsu photomultiplier, using the front face acquisition mode. The excitation 
Submitted to

source was a 450W Xe arc lamp. The emission spectra were corrected for detection and optical spectral response of the spectrofluorimeter, and the excitation spectra were corrected for the spectral distribution of the lamp intensity using a photodiode reference detector. The time-resolved experiments were performed with the setup described for the luminescence spectra using a pulsed Xe-Hg lamp (6 $\mu$ s pulse at half width and 20-30 $\mu$ s tail). The CIE $(\mathrm{x}, \mathrm{y})$ color coordinates were calculated from the emission spectra using the $2^{\text {nd }}$ observer data. A layer of U-1.3 was deposited onto an integrated circuit with tracks of different widths (down to $200 \mu \mathrm{m}$ ), allowing temperature gradients up to 0.03 degree $\mu \mathrm{m}^{-1}$. A translation stage makes possible moving the circuit with a precision of $100 \mu \mathrm{m}$. An optical fiber (spot diameter $\approx 900 \mu \mathrm{m}$ ) is used to illuminate the hybrid layer at $355 \mathrm{~nm}$ and collect the emission spectra, which was converted in absolute temperature.

\section{Acknowledgements}

We acknowledge Fundação para a Ciência e a Tecnologia (FCT, Portugal), COMPETE and FEDER programs (PTDC/CTM/101324/2008) and Integrated Spanish-Portuguese Action PT2009-0131 for financial support. The work in Zaragoza has been supported by the grants MAT2007-61621 and CONSOLIDER CSD2007-00010 from the Ministry of Education. CDSB (SFRH/BD/38472/2007) and PPL (SFRH/BPD/34365/2006) thank FCT for grants. NJOS acknowledges CSIC for a I3P contract. We thank A.L.L. Videira for a critical reading of the manuscript; Carlos Vicente and André Martins (IT-Aveiro) for the help with the tests in the integrated circuit; and Victor Sorribas (Zaragoza) for the collaboration with the optical microscopy observations.

Received: ((will be filled in by the editorial staff))

Revised: ((will be filled in by the editorial staff)) Published online: ((will be filled in by the editorial staff)) 


\section{Submitted to

\section{Figure Captions}

Figure 1. (a) DLS data showing the diameter distribution of the $\gamma-\mathrm{Fe}_{2} \mathrm{O}_{3}$ and $\mathrm{Ln}^{3+}$ doped $\gamma-\mathrm{Fe}_{2} \mathrm{O}_{3} @$ TEOS/APTES nanoparticles, respectively. (b, c) SEM and TEM images, respectively, of NP3-1.3. (d) EDS mappings of the relative Eu and Tb content in the SEM image of NP3-1.3 shown bellow. (e) Image of the optical microscope (UV excitation at $365 \mathrm{~nm}$ ) of NP3-1.10. The red color corresponds to the real color.

Figure 2. (a) Emission spectra of NP3-1.3 excited at $357 \mathrm{~nm}$ and recorded between 14 and $300 \mathrm{~K}$. The sharp lines assigned to $1,2,3,4$ and 5 correspond to the ${ }^{5} \mathrm{D}_{4} \rightarrow{ }^{7} \mathrm{~F}_{6,5}$ $\left(\mathrm{Tb}^{3+}\right)$ and ${ }^{5} \mathrm{D}_{0} \rightarrow{ }^{7} \mathrm{~F}_{2-4}\left(\mathrm{Eu}^{3+}\right)$ transitions, respectively. In the area marked with an asterisk there is an overlap of the $\mathrm{Eu}^{3+}\left({ }^{5} \mathrm{D}_{0} \rightarrow{ }^{7} \mathrm{~F}_{0,1}\right)$ and $\mathrm{Tb}^{3+}\left({ }^{5} \mathrm{D}_{4} \rightarrow{ }^{7} \mathrm{~F}_{4}\right)$ emissions. (b) CIE chromaticity diagram showing the temperature dependence of the $(\mathrm{x}, \mathrm{y})$ color coordinates of NP3-1.3 and U1.3. (c) Temperature dependence of the ${ }^{5} \mathrm{D}_{4}$ lifetime of NP3-1.1, NP3-1.2, NP3-1.3 and U-1.3, monitored at $547 \mathrm{~nm}$ and excited at $357 \mathrm{~nm}$. The lines correspond to the best fit of the experimental data using Eq. (1) $\left(r^{2}>0.983\right)$.

Figure 3. (a) Normalized $\Delta$ parameter (divided by its value at room temperature $\Delta_{0}$ ) of NP3-1.1, NP3-1.2, NP3-1.3, NP3-1.10 (excitation wavelength of $357 \mathrm{~nm}$ ) and U-1.3 (excitation wavelength of $365 \mathrm{~nm}$ ). (b) Sensitivity of NP3-1.1, NP3-1.2, NP3-1.3, NP31.10 and U-1.3. (c) Integrated areas of the ${ }^{5} \mathrm{D}_{4} \rightarrow{ }^{7} \mathrm{~F}_{5}$ (green squares) and ${ }^{5} \mathrm{D}_{0} \rightarrow{ }^{7} \mathrm{~F}_{2}$ (red squares) transitions as a function of temperature for $\mathbf{U}-\mathbf{1 . 3}$ (excitation wavelength of 365 $\mathrm{nm}$ ). The green line corresponds to the best fit of the experimental data using Eq. (1) (correlation coefficient $r^{2}>=0.978$ ) and the red line is a guide for the eyes.

Figure 4. $(a, b)$ Scheme of the temperature measurement using an optical fiber sensing (spot diameter $0.9 \mathrm{~mm}$ ) on an integrated circuit with tracks of different widths (down to $200 \mu \mathrm{m}$ ) covered with a layer of U1.3. (c, d) Temperature profiles obtained with U1.3 (red circles, the size corresponds to the temperature uncertainly of 0.5 degree) along the lines A and B compared with the measurements performed using an IR camera (blue squares). 


\section{ADVANCED}

Figure 1
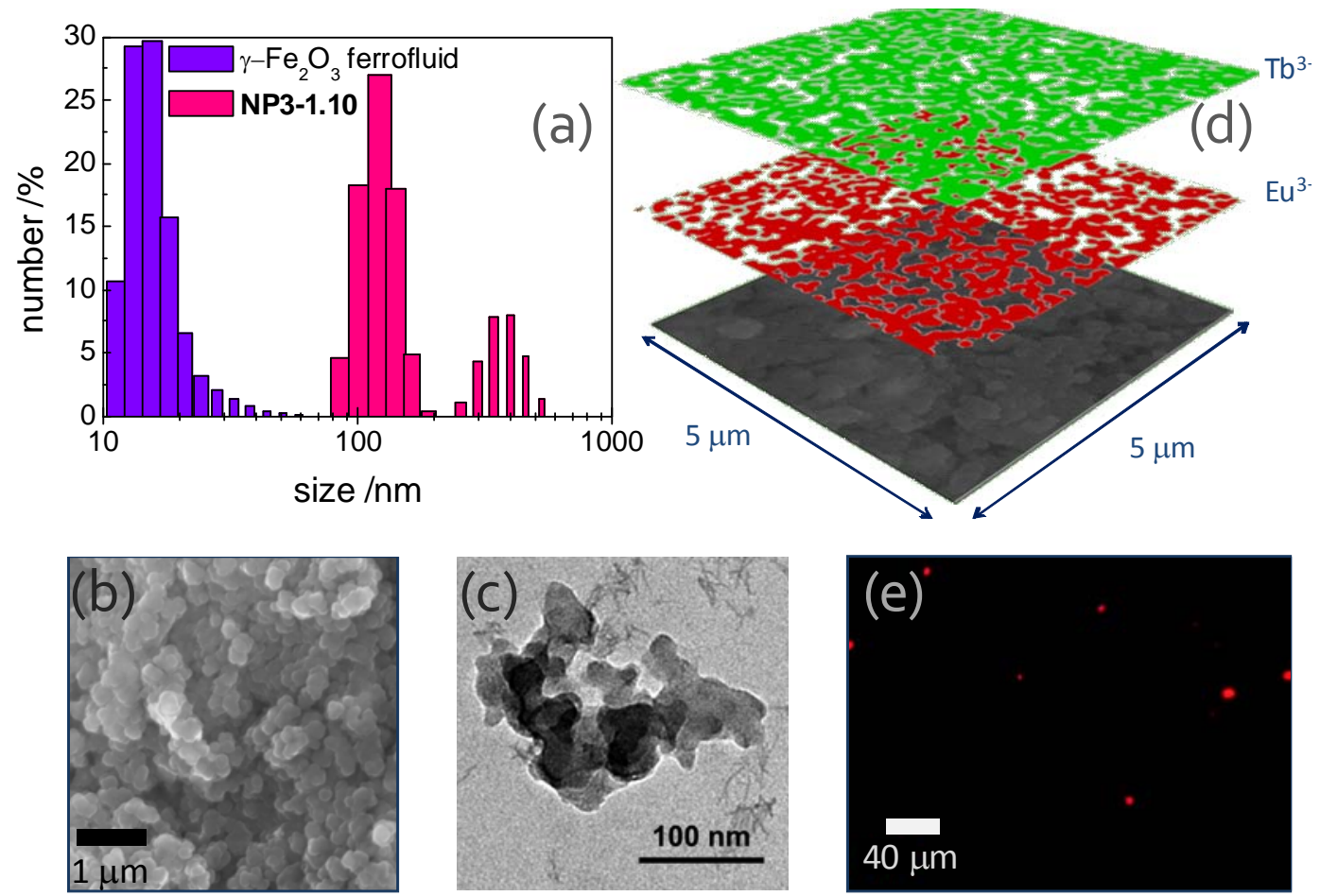
Figure 2

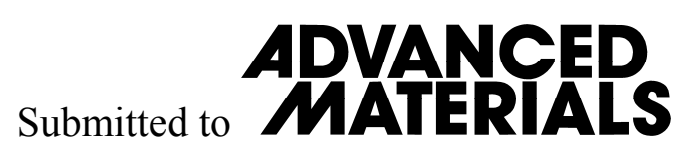

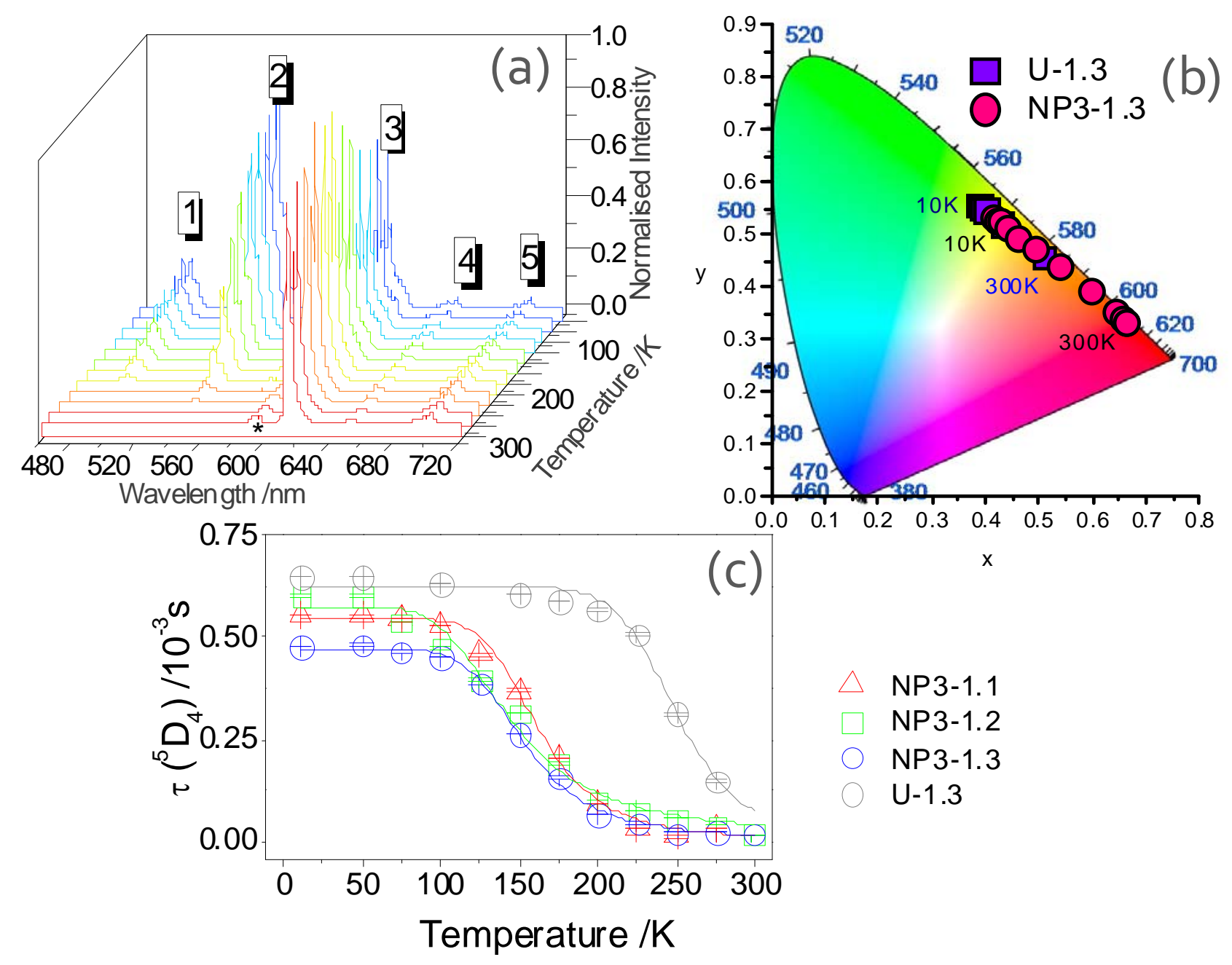


Figure 3

\section{ADVANCED}
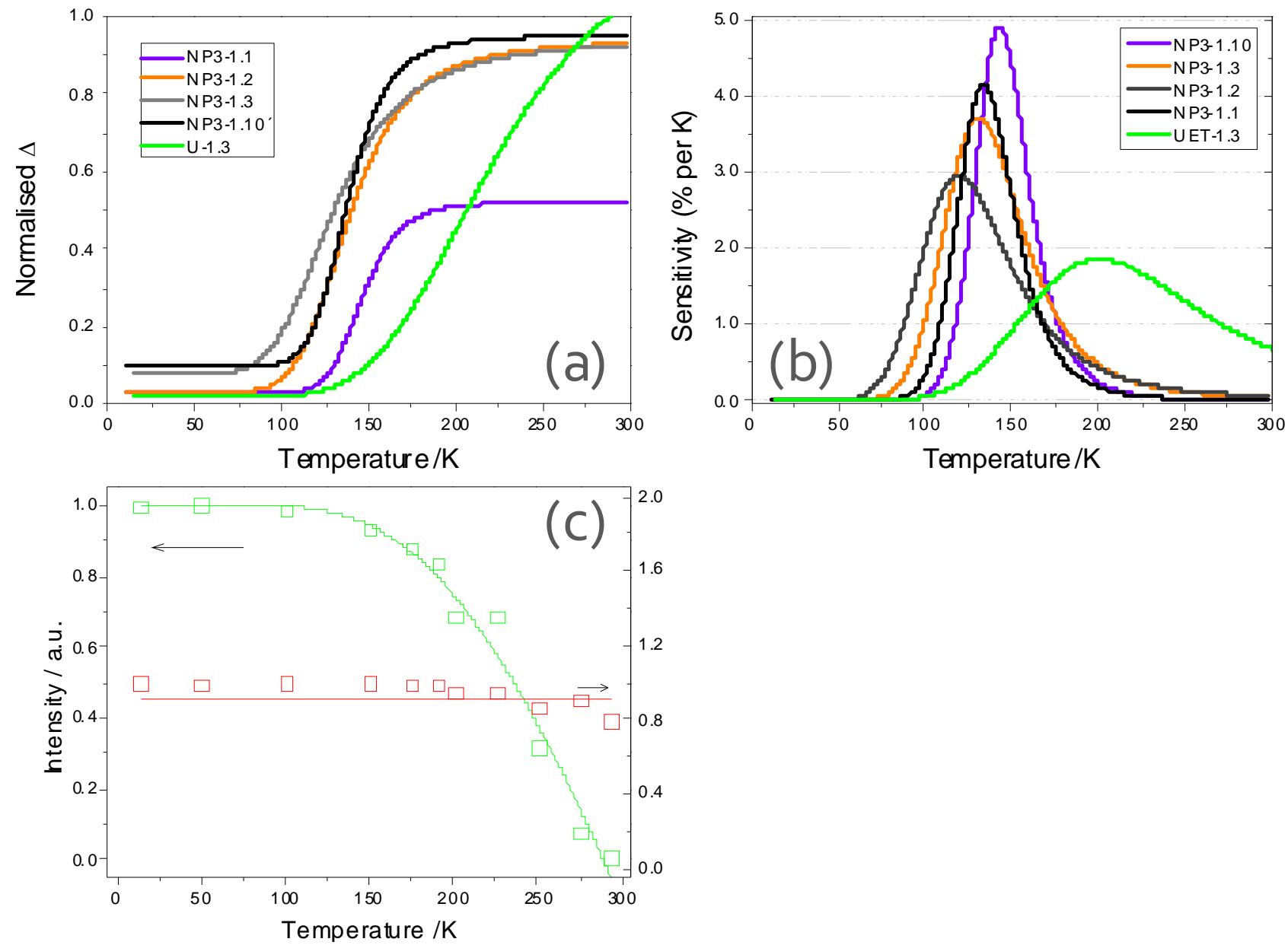


\section{ADVANCED}

Figure 4
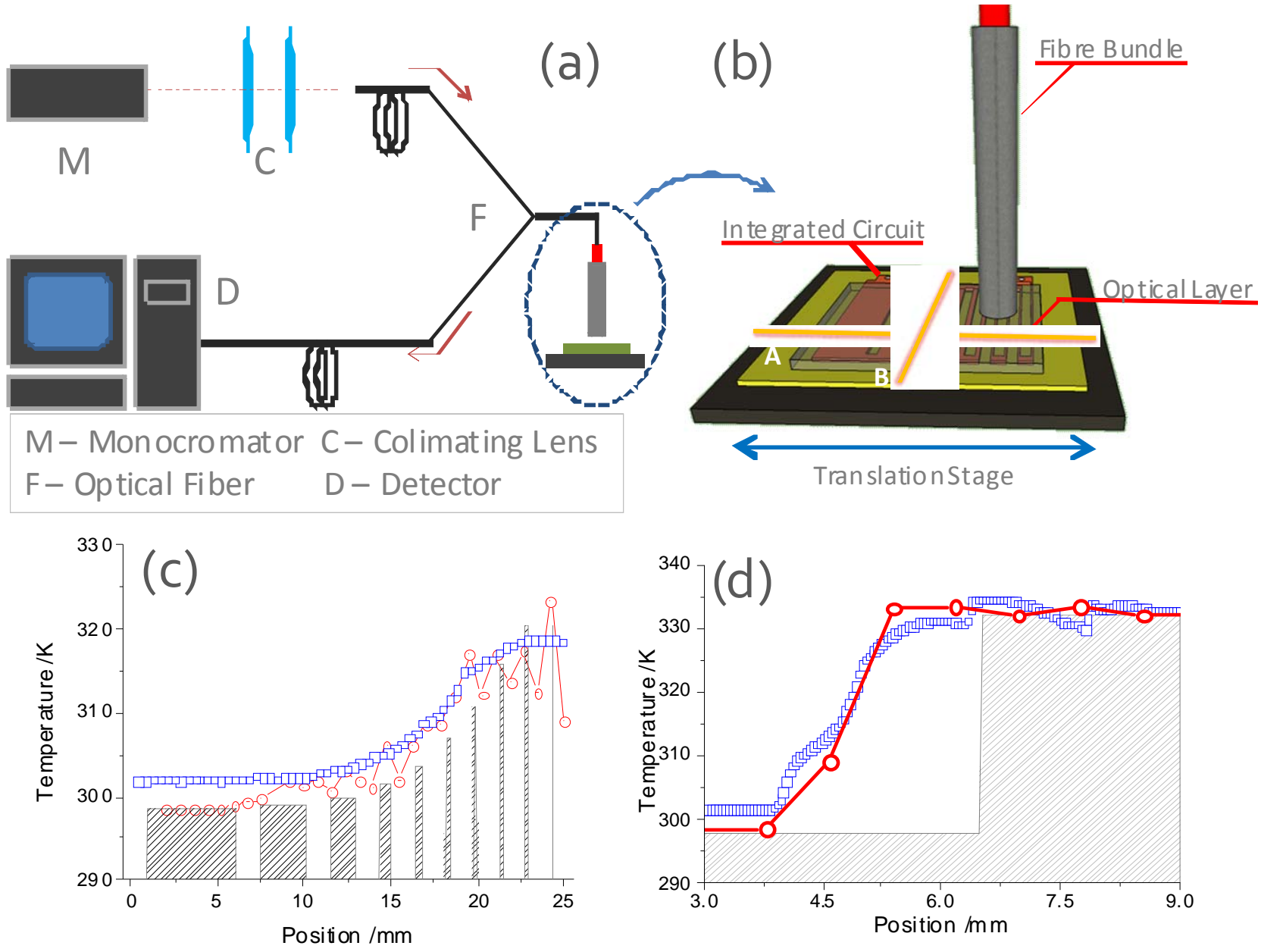
[1] P. R. N. Childs, J. R. Greenwood, C. A. Long, Rev. Sci. Instrum. 2000, 71, 2959.

[2] Y. H. Gao, Y. Bando, Nature 2002, 415, 599.

[3] J. Lee, A. O. Govorov, N. A. Kotov, Angew. Chem. Int. Edit. 2005, 44, 7439.

[4] J. Lee, N. A. Kotov, Nano Today 2007, 2, 48.

[5] P. Löw, B. Kim, N. Takama, C. Bergaud, Small 2008, 4, 908.

[6] E. Saïdi, B. Samson, L. Aigouy, S. Volz, P. Löw, C. Bergaud, M. Mortier, Nanotechnology 2009, 20, 115703.

[7] S. Singh, K. Kumar, S. Rai, Sensor Actuat. A-Phys. 2009, 149, 16.

[8] C. Gota, K. Okabe, T. Funatsu, Y. Harada, S. Uchiyama, J. Am. Chem. Soc. 2009, 131, 2766.

[9] H. S. Peng, M. I. J. Stich, J. B. Yu, L. N. Sun, L. H. Fischer, O. S. Wolfbeis, $A d v$. Mater. 2010, 22, 716.

[10] R. Schorer, E. Friess, K. Eberl, G. Abstreiter, Phys. Rev. B 1991, 44, 1772.

[11] W. Ryba-Romanowski, J. Phys. Chem. Solids 1993, 54, 153.

[12] S. Allison, G. Gillies, Rev. Sci. Instrum. 1997, 68, 2615.

[13] N. Chandrasekharan, L. Kelly, J. Am. Chem. Soc. 2001, 123, 9898.

[14] G. Walker, V. Sundar, C. Rudzinski, A. Wun, M. Bawendi, D. Nocera, Appl. Phys. Lett. 2003, 83, 3555.

[15] S. Allison, G. Gillies, A. Rondinone, M. Cates, Nanotechnology 2003, 14, 859.

[16] B. Zelelow, G. Khalil, G. Phelan, B. Carlson, M. Gouterman, J. Callis, L. Dalton, Sensor Actuat. B-Chem. 2003, 96, 304.

[17] M. Mitsuishi, S. Kikuchi, T. Miyashita, Y. Amao, J. Mater. Chem. 2003, 13, 2875.

[18] G. A. Baker, S. N. Baker, T. M. McCleskey, Chem. Commun. 2003, 2932.

[19] G. E. Khalil, K. Lau, G. D. Phelan, B. Carlson, M. Gouterman, J. B. Callis, L. R. Dalton, Rev. Sci. Instrum. 2004, 75, 192.

[20] A. Heyes, S. Seefeldt, J. Feist, Opt. Laser Technol. 2006, 38, 257.

[21] H. Aizawa, T. Katsumata, S. Komuro, T. Morikawa, H. Ishizawa, E. Toba, Sensor Actuat. A-Phys. 2006, 126, 78.

[22] S. Uchiyama, A. P. de Silva, K. Iwai, J. Chem. Educ. 2006, 83, 720.

[23] X. Wang, X. G. Kong, Y. Yu, Y. J. Sun, H. Zhang, J. Phys. Chem. C 2007, 111, 15119.

[24] S. Katagiri, K. Manseki, Y. Tsukahara, K. Mitsuo, Y. Wada, J. Alloy. Compd. 2008, 453, 1 .

[25] C. Gota, S. Uchiyama, T. Yoshihara, S. Tobita, T. Ohwada, J Phys Chem B 2008, $112,2829$.

[26] A. Khalid, K. Kontis, Meas. Sci. Technol. 2009, 20, 025305.

[27] V. Tikhomirov, K. Driesen, V. Rodriguez, P. Gredin, M. Mortier, V. Moshchalkov, Opt. Express 2009, 17, 11794.

[28] S. Singh, K. Kumar, S. Rai, Appl. Phys. B: Lasers Opt. 2009, 94, 165.

[29] L. Aigouy, E. Saïdi, L. Lalouat, J. Labéguerie-Egéa, M. Mortier, P. Löw, C. Bergaud, J. Appl. Phys. 2009, 106, 4301.

[30] F. Palacio, A. Millán, N. J. Silva, L. D. Carlos, V. Amaral, P. P. Lima, C. D. S. Brites, Spain Patent P200930367, 2009.

[31] P. P. Lima, S. S. Nobre, R. O. Freire, S. A. Junior, R. A. Sa Ferreira, U. Pischel, O. L. Malta, L. D. Carlos, J Phys Chem C 2007, 111, 17627.

[32] L. D. Carlos, R. A. Ferreira, J. P. Rainho, V. de Zea Bermudez, Adv. Funct. Mater. 2002, 12, 819.

[33] A. M. Jakob, T. A. Schmedake, Chem. Mater. 2006, 18, 3173. 


\section{Submitted to

[34] L. Wang, M. C. Estevez, M. O'Donoghue, W. H. Tan, Langmuir 2008, 24, 1635.

[35] V. D. Bermudez, L. D. Carlos, L. Alcacer, Chem. Mater. 1999, 11, 569.

[36] L. D. Carlos, R. A. S. Ferreira, V. D. Bermudez, S. J. L. Ribeiro, Adv. Mater. 2009, 21, 509 .

[37] A. Jordan, R. Scholz, K. Maier-Hauff, M. Johannsen, P. Wust, J. Nadobny, H. Schirra, H. Schmidt, S. Deger, S. Loening, W. Lanksch, R. Felix, J. Magn. Magn. Mater. 2001, 225, 118. 